\title{
Modelos de taper do tipo expoente-forma para descrever o perfil do fuste de árvores
}

\author{
Valdir Carlos Lima de Andrade ${ }^{1}$ \\ ${ }^{1}$ Universidade Federal do Tocantins, Curso de Engenharia Florestal, CEP 77.402-970, Gurupi, TO, Brasil
}

\begin{abstract}
"Autor correspondente:
vclandrade@hotmail.com
\end{abstract}

Termos para indexação:

Multiprodutos madeireiros

Bioestatística florestal

Modelagem florestal

Index terms:

Multiproduct loggers

Forest Biostatistics

Modeling Forest

Histórico do artigo:

Recebido em 11/11/2013

Aprovado em 19/12/2014

Publicado em 31/12/2014

doi: 10.4336/2014.pfb.34.80.614
Resumo - Neste trabalho o objetivo foi avaliar modelos de taper do tipo expoenteforma e outros tipos aplicados no Brasil. Dados de 270 árvores-amostra cubadas do híbrido Eucalyptus urophylla e Eucalyptus grandis foram utilizados como um estudo de caso, avaliando-se 18 modelos de taper dos tipos: simples ( 2 ), biomatemático ( 4 ), segmentado ( 2 ) e expoente-forma ( 10 ). Na avaliação destes modelos, adotouse a análise da distribuição de resíduos e das estatísticas: correlação linear múltipla, erro padrão residual, porcentagem de parcelas não significativas em um delineamento inteiramente casualizado em parcelas subdivididas e erro médio Dunnett, ambos ao nível de 5\% de significância. Concluiu-se que modelos de taper expoentes-forma, de maneira geral, são superiores aos demais tipos de modelos de taper; o modelo segmentado de Clark et al. é superior ao de Max e Burkhart e o modelo biomatemático, desenvolvido neste trabalho, é melhor que os demais modelos biomatemáticos avaliados.

\section{Exponet taper-shape models to describe tree trunks}

Abstract - This study evaluated exponent taper-shape models and other types applied in Brazil. Data from 270 sample trees scaled-hybrid Eucalyptus urophylla and Eucalyptus grandis were used as a studying case with 18 taper types models: simple (2), biomathematics (4), segmented (2) and exponent-form (10). It was adopted the analysis of the residual distribution and statistics: multiple linear correlation, residual standard error, percentage of no significant parcels in a completely randomized split plot and average error Dunnett, both at the level of 5\% significance level. It was concluded that models of taper-shape exponents, in general, are superior to other types, the segmented model of Clark et al. is superior to Max and Burkhart biomathematics and the model developed in this paper, is better than the other biomathematics evaluated. 


\section{Introdução}

A crescente necessidade em otimizar o potencial madeireiro dos povoamentos florestais exige a quantificação das diferentes possibilidades de seu uso que, por conseguinte, exige o emprego de equações de volume, ou de taper, preferencialmente, próprias ao sortimento de multiprodutos da madeira de árvores em pé. Nesta condição, conforme Andrade (2001), o emprego de equações de taper é mais apropriado porque permitem quantificar o volume e o número de toras para diferentes comprimentos em um mesmo diâmetro comercial, ou, para um mesmo comprimento de toras com diferentes diâmetros comerciais.

Equações de taper são geradas a partir da análise de regressão em dados de cubagem rigorosa, geralmente, obtidos em algumas árvores-amostra abatidas. São empregados modelos estatísticos adequados à caracterização da variação de diâmetros do tronco em toda a sua extensão no sentido base-topo, denominados modelos de taper, afilamento, adelgaçamento, perfil, ou, de forma do tronco. Desta caracterização, é possível identificar, facilmente, produtos madeireiros de acordo com informações pré-estabelecidas de diâmetros comerciais e comprimentos de toragens desejados.

Muitos modelos de taper foram desenvolvidos desde a primeira proposta feita pelo Sueco Hojer em 1903. Conforme destacam Ahrens \& Holbert (1981), Pires \& Calegario (2007) e Andrade (2001), até a década de 1960 ocorreram as importantes contribuições de Johson (1911), Behre (1923), Matte (1949), Gray (1956) e Prodan (1965). Da década de 1960 até a atualidade, pode-se citar as contribuições ao estudo sobre taper de Kozak et al. (1969), Demaerschalk (1971), Hradetzky (1976), Max \& Burkhart (1976), Garay (1979), Ahrens \& Holbert (1981), Kozak (1988), Newnham (1988), McTague et al. (1989), Clark et al. (1991), Guimarães \& Leite (1992), Péllico Neto (1994), Angelo et al. (1997), Muhairwe (1999), Andrade \& Leite (2001), Lee et al. (2003), Pires \& Calegario (2007), dentre outros.

Conforme Figueiredo-Filho et al. (1996), modelos de taper foram introduzidos no Brasil a partir da década de 1970 com o trabalho de Silva (1976). Neste ínterim, até a década de 1990, notam-se muitos trabalhos sobre taper no Brasil com ênfase aos modelos de Schoepfer (1966), Kozak et al. (1969), Demaerschalk (1971) e de Ormerod (1973), dentre os quais, pode-se citar os trabalhos de Campos \& Ribeiro (1982), Silva (1982), Schneider (1986), Friedl (1989) e McTague et al. (1989).
A partir da década de 1990 houve um grande aumento nos estudos sobre taper em florestas brasileiras, cabendo citar: Figueiredo-Filho et al. (1996), Schneider et al. (1996), Fischer et al. (2001), Assis et al. (2001), Chichorro et al. (2003), Leite et al. (2006), Mendonça et al. (2007), Souza et al. (2008), Leite et al. (2011), Silva et al. (2011), Soares et al. (2011), Favalessa et al. (2012) e Lanssanova et al. (2013). Nestes trabalhos, além dos modelos citados anteriormente, há destaques aos modelos de Goulding \& Murray (1976), Hradetzky (1976), Max \& Burkhart (1976), Garay (1979), Baldwin Jr. \& Feduccia (1991) e Clark et al. (1991).

Diante dessas considerações, os trabalhos brasileiros englobam uma gama diferenciada de modelos estatísticos para descrever o taper do tipo simples, como por exemplo, modelos de Demaerschalk (1971), Ormerod (1973) e de Hradetzky (1976), segmentados como Goulding \& Murray (1976), Max \& Burkhart (1976) e Clark et al. (1991), polinomiais como os modelos de Kozak et al. (1969) e Schoepfer (1966) e sigmóides como os modelos de Garay (1979), Baldwin Jr. \& Feduccia (1991) e Guimarães \& Leite (1992).

Por outro lado, nota-se que não há trabalhos brasileiros sobre os modelos de taper que se caracterizam pela modelagem de diferentes formas geométricas do tronco de árvores, como por exemplo, os modelos de Kozak (1988), Newnham (1988) e de Muhairwe (1999). Conforme estes autores após aplicar a técnica de regressão stepwise, utiliza-se da regressão não linear para estimar uma equação de taper. Newnham (1999) e Kozak (1988) descrevem este tipo de modelagem, dito modelos de taper expoente-forma, ou variável-forma, ou, ainda variável-expoente, sendo denominados neste trabalho de: modelos de taper expoente-forma.

Portanto, desenvolveu-se o presente trabalho tendo como objetivo avaliar diferentes modelos de taper já existentes e avaliados no Brasil, bem como, desenvolver modelos de característica biomatemática e do tipo expoente-forma.

\section{Material e métodos}

Os dados utilizados como um estudo de caso foram obtidos em plantios comerciais de Eucalyptus urophylla e Eucalyptus grandis localizados na região nordeste do Estado da Bahia, com idades variando entre 5 a 7 anos. Foram cubadas 270 árvores-amostra com medições de diâmetros ao longo do tronco nas posições: $0,3 \mathrm{~m}, 0,7 \mathrm{~m}$, 
$1,3 \mathrm{~m}, 2,0 \mathrm{~m}$ e, deste ponto em diante, a cada 2,0 $\mathrm{m}$ até um diâmetro em torno de 2,0 cm com casca.

Dados de 90 árvores-amostra, com dez indivíduos por classe de diâmetro de $2,0 \mathrm{~cm}$ cada, foram utilizados no ajuste de vários modelos de taper. O restante dos dados, com 180 árvores-amostra, foi reservado para um teste de aplicação visando avaliar a predição das equações de taper geradas. Assim, utilizando os dados da cubagem de 90 árvores-amostra, procedeu-se ao ajuste dos seguintes modelos de taper:

$$
\begin{aligned}
& \left(\frac{d_{i}}{d}\right)=(10)^{\beta_{0}}(d)^{\left(\beta_{1}-1\right)}\left(h-h_{i}\right)^{\beta_{2}}(h)^{\beta_{3}}+\varepsilon \\
& \left(\frac{d_{i}}{d}\right)=\beta_{0}\left(\frac{h-h_{i}}{h-1,3}\right)^{\beta_{1}}+\varepsilon \\
& \left(\frac{d_{i}}{d}\right)=\beta_{0}\left\{1+\beta_{1} \operatorname{Ln}\left[1-\beta_{2}\left(\frac{h_{i}}{h}\right)^{\beta_{3}}\right]\right\}+\varepsilon \\
& \left(\frac{d_{i}}{d}\right)=\beta_{1}+\beta_{2} \operatorname{Ln}\left\{1-\left(\frac{h_{i}}{h}\right)^{\left(\frac{1}{m}\right)}\left[1-\exp \left(-\frac{\beta_{1}}{\beta_{2}}\right)\right]\right\}+\varepsilon \\
& \left(\frac{d_{i}}{d}\right)=\left\{\exp \left[1-\exp \left(1,3-h_{i}\right)\right]\right\}^{\beta_{1}}\left(\frac{h-h_{i}}{h-1,3}\right)^{\beta_{2}}+\varepsilon \\
& \left.\left(\frac{d_{i}}{d}\right)=\left(\frac{h-h_{i}}{h-1,3}\right)\left\{1 / \beta_{0}+\beta_{1}\left(\frac{d}{h}\right)+\beta_{2}\left(\frac{d}{h}\right)^{2}+\beta_{3}\left(\frac{1}{h_{i}}\right)\right]\right\}+\varepsilon \\
& \left.\left(\frac{d_{i}}{d}\right)=\left(\frac{h-h_{i}}{h-1,3}\right)\left\{1 / \beta_{0}+\beta_{1}\left(\frac{h-h_{i}}{h-1,3}\right)^{6}+\beta_{2}\left(\frac{d}{h}\right)+\beta_{3}\left(\frac{h-h_{i}}{h-1,3}\right)^{2}\left(\frac{d}{h}\right)\right]\right\}+\varepsilon \\
& \left(d_{i}\right)=a_{0} d^{a_{1}} a_{2}^{d}\left[\frac{\left(1-\sqrt{\frac{h_{i}}{h}}\right)}{(1-\sqrt{P})}\right]^{\left.\beta_{1}\left(\frac{h_{i}}{h}\right)^{2}+\beta_{2} \operatorname{Ln}\left(\frac{h_{i}}{h}+0,001\right)+\beta_{3} \sqrt{\frac{h_{i}}{h}}+\beta_{4} \exp \left(\frac{h_{i}}{h}\right)+\beta_{5}\left(\frac{d}{h}\right)\right]}+\varepsilon
\end{aligned}
$$


$\left(d_{i}\right)=a_{0} d^{a_{1}} a_{2}^{d}\left(1-\sqrt{\frac{h_{i}}{h}}\right)^{\left[\beta_{1}\left(\frac{h_{i}}{h}\right)^{2}+\beta_{2}\left(\frac{h}{h_{i}}\right)+\beta_{3}(d)+\beta_{4}(h)+\beta_{5}\left(\frac{d}{h}\right)\right]}+\varepsilon$

$\left(d_{i}\right)=d\left[\beta_{1}\left(\frac{h_{i}}{h}-1\right)+\beta_{2}\left({\frac{h_{i}}{h}}^{2}-1\right)+\beta_{3}\left(a_{1}-\frac{h_{i}}{h}\right)^{2} I_{1}+\beta_{4}\left(a_{2}-\frac{h_{i}}{h}\right)^{2} I_{2}\right]^{0,5}+\varepsilon$

e

$$
\left(d_{i}\right)=\left\{\begin{array}{l}
\frac{I_{S} d^{2}\left[1+\left(\beta_{1}+\frac{\beta_{2}}{d^{3}}\right)\left(\left(1-\frac{h_{i}}{h}\right)^{\beta_{3}}-\left(1-\frac{1,3}{h}\right)^{\beta_{3}}\right]\right.}{1-\left(1-\frac{1,3}{h}\right)^{\beta_{3}}\left[\frac{d^{2}-\left(d^{2}-d_{5,3}^{2}\right)\left(\left(1-\frac{1,3}{h}\right)^{\beta_{4}}-\left(1-\frac{h_{i}}{h}\right)^{\beta_{4}}\right]}{\left.\left(1-\frac{1,3}{h}\right)^{\beta_{4}}-\left(1-\frac{5,3}{h}\right)^{\beta_{4}}\right]}\right]} \\
+I_{T}\left[d_{5,3}^{2}\left(\beta_{5}\left(\frac{h_{i}-5,3}{h-5,3}-1\right)^{2}+I_{M}\left(\frac{1-\beta_{5}}{\beta_{6}^{2}}\right)\left(\beta_{6}-\frac{h_{i}-5,3}{h-5,3}\right)^{2}\right)\right]
\end{array}\right\}+\varepsilon
$$

Onde: $\mathrm{d}=$ DAP $(\mathrm{cm}), \mathrm{h}=$ altura total $(\mathrm{m}), \mathrm{d}_{\mathrm{i}}=$ diâmetro com casca medido ao longo do tronco $(\mathrm{cm}), \mathrm{h}_{\mathrm{i}}=$ altura onde se mediu $\mathrm{d}_{\mathrm{i}}(\mathrm{m}), \mathrm{d}_{5,3}=$ diâmetro do tronco com casca situado à $5,3 \mathrm{~m}(\mathrm{~cm}), \mathrm{P}=$ ponto de inflexão, exp = inverso do logaritmo neperiano, $\mathrm{a}_{0}, \mathrm{a}_{1}, \mathrm{a}_{2}, \beta_{0}, \ldots, \beta_{6}=$ parâmetros à estimar por regressão não linear, $\varepsilon=$ erro aleatório,

$$
\begin{aligned}
& I_{i}=\left[\begin{array}{c}
1 s e \frac{h_{i}}{h} \leq a_{1} \\
0 s e \frac{h_{i}}{h}>a_{1}
\end{array}\right], \quad I_{S}=\left[\begin{array}{c}
1 \operatorname{seh}_{i}<1,3 \\
0 \operatorname{seh}_{i} \geq 1,3
\end{array}\right], \\
& I_{B}=\left[\begin{array}{l}
1 \text { se } 1,3 \leq h_{i} \leq 5,3 \\
0 \text { Se diferente da condição acima }
\end{array}\right] \text {, } \\
& I_{T}=\left[\begin{array}{l}
1 \operatorname{seh}_{i}>5,3 \\
0 \operatorname{seh}_{i} \leq 5,3
\end{array}\right], \quad \text { e } \\
& I_{M}=\left[\begin{array}{l}
1 \operatorname{se~}_{i}<\left(5,3+{ }_{1}\left({ }_{i}-5,3\right)\right) \\
0 \text { Se diferente da condição acima }
\end{array}\right] \text {. }
\end{aligned}
$$

Os modelos 1 e 2, respectivamente, de Demaerschalk (1971) e Ormerod (1973), são os modelos simples respectivamente, modelos de Garay (1979), Baldwin Jr. \& Feduccia (1991) e Guimarães \& Leite (1992), são os mais utilizados no Brasil. Já, os modelos 3, 4 e 5, modelos de taper biomatemáticos recentemente testados no 
Brasil, conforme estudos conduzidos por Leite et al. (2006), Silva et al. (2011), Leite et al. (2011) e Soares et al. (2011).

Os modelos 6, 7, 8 e 9, respectivamente, modelos de Newhan (1988), Kozak (1988) e Muhairwe (1999), foram escolhidos por serem modelos de taper do tipo expoenteforma ainda não testados em povoamentos florestais brasileiros. Por último, os modelos 10 e 11, respectivamente, de Max \& Burkhart (1976) e Clark et. all (1991), foram escolhidos por serem modelos de taper segmentados já testados no Brasil, por exemplo, por Figueiredo-Filho et al. (1996), Assis et al. (2001), Souza et al. (2008), Favalessa et al. (2012) e Lanssanova et al. (2013).

Ressalta-se que, no modelo de Baldwin Jr. \& Feducia (1991), foram realizadas três formas de ajuste adotando-se $m=3, m=\beta_{3}$ e variando-se unidades a partir de $m=2$ para obter a melhor opção de estimativa deste coeficiente. Já, no modelo de Kozak (1988), também foram realizadas três formas de ajuste para obter P, quais sejam: valor de $\mathrm{P}$ conforme originalmente feito pelo autor, valor de $\mathrm{P}$ sendo assumido como um parâmetro do modelo a ser estimado $(\hat{P})$ e valor fixo de $\mathrm{P}$, obtido pela variação a cada 0,05 a partir de 0,1 . Também, a partir de adaptações do modelo de Chapman-Richards, desenvolveu-se o seguinte modelo de taper biomatemático, ou, do tipo sigmóide:

$$
\left(\frac{d_{i}}{d}\right)=\left\{1-\left(\frac{h_{i}-1,3}{h-1,3}\right) \exp \left[-\beta_{1}\left(1-\frac{h_{i}}{h}\right)^{\beta_{2}}\right]\right\}^{\beta_{3}}+\varepsilon
$$

Ainda, baseando - se em Ormerod (1973), Kozak(1988) e Newnhan (1988), a partir de :

$$
\begin{aligned}
& \left(\frac{d_{i}}{d}\right)=\left(\frac{h-h_{i}}{h-1,3}\right)^{c_{1}}, \\
& \left(\frac{d_{i}}{d}\right)=\left(\frac{h-h_{i}}{h-1,3}\right) \\
& \left(\frac{d_{i}}{c_{2}}\right)=\left[1-\left(\frac{h_{i}-1,3}{h-1,3}\right)\right]^{c_{3}},
\end{aligned}
$$

respectivamente, obteve - se :

$$
\begin{gathered}
c_{1}=\frac{\operatorname{Ln}\left(\frac{d_{i}}{d}\right)}{\operatorname{Ln}\left(\frac{h-h_{i}}{h-1,3}\right)}, \\
c_{2}=\frac{\operatorname{Ln}\left(\frac{h-h_{i}}{h-1,3}\right)}{\operatorname{Ln}\left(\frac{d_{i}}{d}\right)} \mathrm{e}^{\prime} \\
c_{3}=\frac{\operatorname{Ln}\left(\frac{d_{i}}{d}\right)}{\operatorname{Ln}\left[1-\left(\frac{h_{i}-1,3}{h-1,3}\right)\right]} .
\end{gathered}
$$


Em seguida, adotando-se o procedimento de regressão Stepwise no relacionamento de $c_{1}, c_{2}$ e $c_{3}$ transformações entre $\mathrm{d}, \mathrm{h}, \mathrm{h}_{\mathrm{i}} \mathrm{e}$ aquelas em que se usam no ajuste do modelo de Hradetzky (1976), desenvolveu-se com diferentes variáveis independentes, obtidas por os seguintes modelos de taper do tipo expoente-forma:

$\left(\frac{d_{i}}{d}\right)=\left(\frac{h-h_{i}}{h-1,3}\right)\left[\beta_{1}\left(\frac{h_{i}}{h}\right)+\beta_{2}\left(\frac{d}{h}\right)+\beta_{3}\left(\frac{d}{h_{i}}\right)+\beta_{4}\left(\frac{h}{\sqrt{h_{i}}}\right)+\beta_{5}\left(\frac{d}{h / h_{i}}\right)+\beta_{6}\left(\frac{h^{2}}{\sqrt{h_{i}}}\right)+\beta_{7}(l)\right]+\varepsilon$

$\left(\frac{d_{i}}{d}\right)=\left(\frac{h-h_{i}}{h-1,3}\right)\left[\beta_{0}+\beta_{1}\left(\frac{d}{h}\right)+\beta_{2}\left(\frac{d}{h}\right)^{2}+\beta_{3}\left(\frac{1}{h_{i}}\right)\right]+\varepsilon$

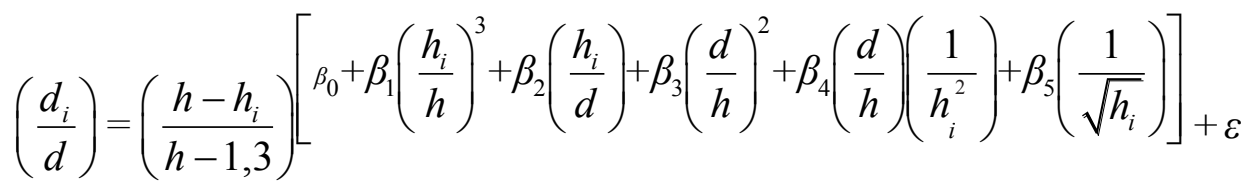

$\left(\frac{d_{i}}{d}\right)=\left(\frac{h-h_{i}}{h-1,3}\right)$

$$
\left.\left\{\begin{array}{l}
\left.1 / \beta_{0}+\beta_{1}\left(\frac{d}{h}\right)+\beta_{2}\left(\frac{1}{h_{i}}\right)^{2}+\beta_{3}\left(\frac{1}{h_{i}}\right)+\beta_{4}\left(\frac{d}{h}\right)\left(\frac{1}{h_{i}}\right)+\beta_{5}\left(\frac{d}{h}\right)^{2}+\beta_{6}\left(\frac{h-h_{i}}{h-1,3}\right)^{3}\left(\frac{d}{h}\right)+\right] \\
\beta_{7}\left(\frac{d}{h}\right)\left(\frac{1}{h_{i}}\right)^{2}+\beta_{8}\left(\frac{h-h_{i}}{h-1,3}\right)^{3}
\end{array}\right]\right\}
$$

$\left(\frac{d_{i}}{d}\right)=\left(1-\frac{h_{i}-1,3}{h-1,3}\right)\left[\beta_{0}+\beta_{1}\left(\frac{h_{i}}{h}\right)^{3}+\beta_{2}\left(h_{i}\right)^{5}+\beta_{3}\left(h_{i}\right)^{3}+\beta_{4}\left(\frac{h_{i}}{d}\right)+\beta_{5}\left(\frac{1}{\sqrt{h_{i}}}\right)\right]+\varepsilon$

$\left(\frac{d_{i}}{d}\right)=\left(1-\frac{h_{i}-1,3}{h-1,3}\right)\left[\beta_{0}+\beta_{1}\left(\frac{d}{h_{i}}\right)+\beta_{2}\left(\frac{d}{h}\right)^{2}+\beta_{3}\left(\frac{h_{i}}{h}\right)^{0,6}+\beta_{4}\left(\frac{h_{i}}{h}\right)^{15}+\beta_{5}\left(\frac{h_{i}}{h}\right)^{90}\right]+\varepsilon$ 
Após o ajuste dos 18 modelos apresentados anteriormente, procedeu-se a uma avaliação preliminar das equações geradas quanto à predição do taper nas 160 árvores-amostra reservadas para este fim, contendo dados independentes das 90 árvores-amostra utilizadas no ajuste. Nesta avaliação preliminar, adotou-se a análise da distribuição de resíduos e das seguintes estatísticas:

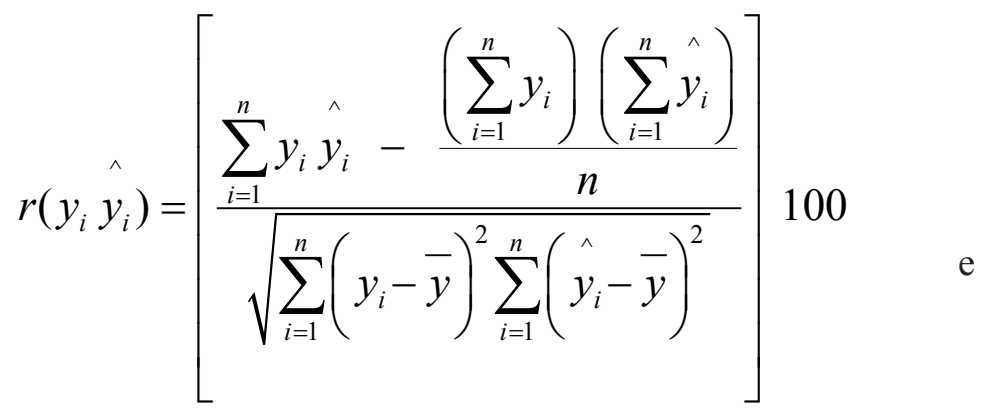

$$
\begin{aligned}
& s\left(y_{i} \hat{y_{i}}\right)= \pm \frac{\sqrt{\frac{\sum_{i=1}^{n}\left(y_{i}-\hat{y_{i}}\right)^{2}}{(n-2)}}}{\bar{y}} 100
\end{aligned}
$$

Onde: $r\left(y_{i} y_{i}\right)=$ correlação linear múltipla entre $y_{i} e^{y_{i}}, s\left(y_{i} y_{i}\right)=$ erro padrão residual

$(\%), y_{i}$ e $y_{i}=$ diâmetro real e predito ao longo do tronco, respectivamente, $\mathrm{n}=$ número de pares de $\mathrm{y}_{\mathrm{i}}$ e $\hat{\mathrm{y}}_{\mathrm{i}}$ e $\overline{\mathrm{y}}=$ média do diâmetro real ao longo do tronco.
Ainda, para uma decisão final quanto aos modelos de taper pré-selecionados, incluiu-se na análise, um delineamento inteiramente casualizado em parcelas subdivididas $\left(\mathrm{DIC}_{\mathrm{sub}}\right)$, conforme estrutura de análise estatística apresentada em Banzatto \& Kronka (2006) e Pimentel-Gomes (2009). Neste experimento em DIC sub' $_{\text {, }}$ as parcelas se referiram às classes de diâmetro e altura com cinco repetições cada (árvores) e os modelos avaliados, incluindo-se o valor real, foram considerados como sendo tratamentos, ou, subparcelas.

No caso em que o teste $\mathrm{F}$ resultou em significância, isto é, caso este resultado representar diferença entre modelos e valor real, escolheu-se recorrer ao teste Dunnett que, conforme Banzatto \& Kronka (2006), permite comparar tratamentos com a testemunha, considerado nesta oportunidade como sendo a média do diâmetro real medido ao longo do tronco das 180 árvores-amostra, dados independentes das 90 árvoresamostra utilizadas para gerar equações de taper.

Diante do resultado do experimento em $\mathrm{DIC}_{\mathrm{sub}}$, considerou-se a porcentagem de parcelas não significativas $\left(\mathrm{P}^{\text {ns }}\right)$ e o erro médio Dunnett (EMD), ponderado pelo número de posições do tronco amostrados por parcela. Incluiu-se, também, com $\mathrm{P}^{\text {ns }}$ e EMD, as estatísticas $r\left(y_{i} \hat{y}_{i}\right)$ e $s\left(y_{i} \hat{y_{i}}\right)$ em um único percentual médio (UPM), obtido por:

$U P M=\frac{\left(100-P^{n s}\right)+E M D+\left[100-r\left(y_{i} \hat{y_{i}}\right)\right]+s\left(y_{i} \hat{y_{i}}\right)}{4}$ 


\section{Resultados e discussão}

Os resultados obtidos para os modelos de 1 à 5 e 12 são apresentados na Tabela 1. Nota-se que o modelo 12 (desenvolvido neste trabalho) e o de Guimarães \& Leite (1992), apresentaram os melhores resultados de $r\left(y_{i} y_{i}\right)$ e $s\left(y_{i} y_{i}\right)$, isto é, resultaram em maior e menor valores, respectivamente. Também, para inferir quanto ao modelo de taper mais difundido no Brasil, o modelo 1, modelo de Demaerschalk (1971) foi selecionado frente ao modelo 2, modelo de Ormerod (1973).

Tabela 1. Parâmetros estimados e resultados dos critérios estatísticos de análise obtidos para os modelos de taper de 1 a 5 e 12 .

\begin{tabular}{ccccccc}
\hline Modelo & $\hat{\beta_{0}}$ & $\hat{\beta_{1}}$ & $\hat{\beta}_{2}$ & $\hat{\beta_{3}}$ & $r\left(y_{i} \hat{y}_{i}\right)$ & $s\left(y_{i} y_{i}\right)$ \\
\hline 1 & 0,0952 & 0,8967 & 0,7152 & $-0,6757$ & 97,80 & 6,95 \\
2 & 1,0203 & 0,7206 & - & - & 97,78 & 6,98 \\
3 & 3,3100 & 0,0840 & 0,999995 & 0,000095 & 98,74 & 5,25 \\
4 & - & 1,4166 & 0,3306 & $8^{*}$ & 98,83 & 5,07 \\
5 & - & $-0,0629$ & 0,6144 & - & 99,00 & 4,69 \\
12 & $-1,6120$ & 6,6670 & 0,6711 & - & 99,02 & 4,63 \\
\hline
\end{tabular}

*valor de m que minimizou $s\left(y_{i} y_{i}\right)$

Na Tabela 2 são apresentados os resultados obtidos para os modelos de 6 a 11, que são os modelos expoenteforma de Newhan (1988), Kozak (1988) e Muhairwe (1999), e modelos segmentados de Max \& Burkhart (1976) e de Clark et al. (1991). Comparando-se com os modelos 5 e 12, nota-se que apenas os modelos 6 e 10 foram piores. Assim, por apresentarem $r\left(y_{i} y_{i}\right) \geq 99,0 \%$ e $s\left(y_{i} y_{i}\right) \leq 5,0 \%$, dentre os modelos de 1 a 12 , os modelos $5,7,8,9,11$ e 12 , previamente, foram selecionados como os seis melhores modelos de taper.

Tabela 2. Parâmetros estimados e resultados dos critérios estatísticos de análise obtidos para os modelos de taper expoenteforma de 6 à 9 e modelos de taper segmentados 10 e 11 .

\begin{tabular}{ccccccc}
\hline \multirow{2}{*}{ Estatísticas } & \multicolumn{7}{c}{ Modelo } \\
\cline { 2 - 7 } & $\mathbf{6}$ & $\mathbf{7}$ & $\mathbf{8}$ & $\mathbf{9}$ & $\mathbf{1 0}$ & $\mathbf{1 1}$ \\
\hline$\hat{a}_{0}$ & - & - & 1,1005 & 1,0375 & - & - \\
$\hat{a}_{1}$ & - & - & 0,9333 & 1,0391 & 0,1173 & - \\
$\hat{a}_{2}$ & - & - & 0,9985 & 0,9928 & 0,8551 & - \\
$\hat{\beta}_{0}$ & 2,5150 & 2,3093 & $0,1528^{*}$ & - & - & - \\
$\hat{\beta}_{1}$ & $-2,3184$ & $-0,5093$ & 0,1331 & 0,2077 & $-4,2300$ & 0,4163 \\
$\hat{\beta}_{2}$ & 1,1566 & $-1,1181$ & $-0,0840$ & $-0,0171$ & 2,0072 & 161,5000 \\
$\hat{\beta}_{3}$ & 0,3274 & $-0,2134$ & $-0,0470$ & $-0,0167$ & 28,6553 & 24,5916 \\
$\hat{\beta}_{4}$ & - & - & 0,1366 & 0,0117 & $-1,7033$ & 6,1661 \\
$\hat{\beta}_{5}$ & - & - & 0,2333 & 0,5264 & - & 3,6689 \\
$\hat{\beta}_{6}$ & - & - & - & - & - & 0,8839 \\
$r\left(y_{i} y_{i}\right)$ & 98,80 & 99,00 & 99,16 & 99,10 & 98,97 & 99,10 \\
$\left.\hat{n}_{i} \hat{y}_{i}\right)$ & 5,12 & 4,68 & 4,30 & 4,43 & 4,76 & 4,43 \\
\hline
\end{tabular}

* refere-se ao valor de $P$ que minimizou $s\left(y_{i} y_{i}\right)$ 
Na Tabela 3 são apresentados os resultados obtidos para os modelos de 13 a 18, que são os modelos expoenteforma desenvolvidos neste trabalho. Comparando-se com as Tabelas anteriores, nota-se que todos estes modelos atendem à exigência de $r\left(y_{i} \hat{y_{i}}\right) \geq 99,0 \%$ e $s\left(y_{i} y_{i}\right) \leq 5,0 \%$. Por isso, estes modelos foram incluídos aos já previamente selecionados, totalizando 13 modelos potenciais para descrever o perfil do tronco de Eucalyptus urophylla e Eucalyptus grandis no nordeste da Bahia com idades variando entre 5 a 7 anos.
Diante disso, para decidir pelo modelo de taper mais adequado dentre os modelos: 1, 5 e 12, 7, 8, 9 e 11 e 13 a 18, pré-selecionados com base na análise da distribuição de resíduos e das estatísticas $r\left(y_{i} \hat{y}_{i}\right)$ e $s\left(y_{i} y_{i}\right)$, foi adotada à análise de um experimento em $\mathrm{DIC}_{\text {sub }}$ onde obteve-se significância para parcelas, modelos e interação entre parcelas e modelos. Com este resultado, foi necessário aplicar o teste Dunnett para verificar diferenças de tratamentos dentro das parcelas com o valor real, considerado como testemunha, ou, comparador.

Tabela 3. Parâmetros estimados e resultados dos critérios estatísticos de análise obtidos para os modelos propostos de taper expoente-forma de 13 à 18 .

\begin{tabular}{ccccccc}
\hline \multirow{2}{*}{ Estatísticas } & \multicolumn{7}{c}{ Modelo } \\
\cline { 2 - 7 } & $\mathbf{1 3}$ & $\mathbf{1 4}$ & $\mathbf{1 5}$ & $\mathbf{1 6}$ & $\mathbf{1 7}$ & $\mathbf{1 8}$ \\
\hline$\hat{\beta}_{0}$ & 0,5719 & - & - & 2,9496 & $-2,4475$ & $-3,7668$ \\
$\hat{\beta}_{1}$ & $-0,0090$ & $-0,0388$ & 0,5728 & $-2,8415$ & 7,4807 & 11,0806 \\
$\hat{\beta}_{2}$ & 0,00085 & 0,445 & 0,1216 & 0,619 & 0,5039 & 0,5115 \\
$\hat{\beta}_{3}$ & $-0,0427$ & $-0,0147$ & 0,0615 & $-3,0029$ & $-0,0037$ & $1,083 \mathrm{E}-8$ \\
$\hat{\beta}_{4}$ & 0,2358 & 0,1123 & - & 3,4598 & 0,1691 & 0,0220 \\
$\hat{\beta}_{5}$ & - & 0,1027 & - & 1,1814 & $-0,3884$ & 0,2076 \\
$\hat{\beta}_{6}$ & - & $-0,00114$ & - & $-2,0905$ & $-0,0028$ & $-0,8447$ \\
$\hat{\beta}_{7}$ & - & - & - & $-0,7225$ & - & - \\
$\hat{\beta}_{8}$ & - & - & - & 1,0313 & - & - \\
$r\left(y_{i} y_{i}\right)$ & 99,16 & 99,02 & 99,07 & 99,12 & 99,09 & 99,06 \\
$s\left(y_{i} y_{i}\right)$ & 4,29 & 4,63 & 4,51 & 4,38 & 4,46 & 4,55 \\
\hline
\end{tabular}

A análise da Tabela 4 mostra que os três melhores modelos, em sequência, são os modelos de taper expoente-forma: 9, 14 e 8, além do modelo 1 em sexto lugar, considerado como a forma usual de descrever o taper de árvores no Brasil. Nos modelos 9 e 14 se observam que $77,78 \%$ das parcelas resultaram em não significância pelo teste Dunnett $\left(\mathrm{P}^{\mathrm{ns}}\right)$ e um erro médio ponderado Dunnett de $0,03 \%$ e $0,12 \%$, respectivamente. Ao incluir estes resultados em um único percentual médio com as estatísticas $r\left(y_{i} \hat{y_{i}}\right)$ e $s\left(y_{i} \hat{y_{i}}\right)$, o resultado foi de $6,89 \%$ e $6,99 \%$, respectivamente. Além destes, o modelo 8 também se mostrou apropriado para caracterizar o taper de eucalipto, se classificando em terceiro lugar.
Nas diferentes formas de análise da distribuição de resíduos para os modelos 1, 8, 9 e 14, apresentadas na Figura 1, nota-se que o modelo 1 foi, expressivamente, pior e os demais modelos de taper tiveram uma dispersão, aproximadamente, igual com resíduos tendo a maioria se dispersando em $\pm 1 \mathrm{~cm}$ (Figuras 1.a e 1.b versus Figuras 1.e, 1.f, 1.i, 1.j, 1.m, 1.n) e com melhor dispersão em torno da reta com $45^{\circ}$ (Figura 1.c versus Figuras 1.g, 1.k e 1.o). De acordo com isto, está o histograma dos resíduos porcentuais (Figura 1.d versus Figuras 1.h, 1.1 e 1.p), onde a maioria dos resíduos oscilam em $\pm 5 \%$, sendo melhor para o modelo 14 . 
Tabela 4. Resultados obtidos no delineamento inteiramente casualizado em parcelas subdivididas aplicando-se o teste Dunnett para avaliar 13 modelos de taper, previamente selecionados dentre um total de 18.

\begin{tabular}{cccccccccccccc}
\hline Modelo & P1 & P2 & P3 & P4 & P5 & P6 & P7 & P8 & P9 & EMD & Pns & UPM & \\
\hline $\mathbf{1}$ & 0 & 0 & 0 & 0 & $-1,45$ & 0 & $-1,01$ & 0 & 1,06 & $-0,141$ & 66,67 & 10,66 & 6 \\
5 & $-4,41$ & $-1,30$ & $-2,56$ & 0 & $-3,02$ & 1,80 & 0 & 1,10 & 2,55 & $-0,416$ & 22,22 & 20,97 & 13 \\
7 & 0 & 0 & 0 & 0 & $-1,74$ & 0 & $-1,29$ & $-1,01$ & 0 & $-0,486$ & 66,67 & 9,87 & 5 \\
$\mathbf{8}$ & 0 & 0 & 0 & 1,07 & 0 & 1,01 & $-0,81$ & 0 & 0 & 0,126 & 66,67 & 9,65 & 3 \\
$\mathbf{9}$ & 0 & 0 & 0 & 0 & 0 & 0 & $-0,82$ & 0 & 0,91 & 0,032 & 77,78 & 6,89 & 1 \\
11 & $-2,93$ & 0 & 0 & 0 & $-1,96$ & 0 & $-1,30$ & 0 & 1,11 & $-0,615$ & 44,44 & 15,37 & 10 \\
12 & $-3,46$ & 0 & 0 & 0 & $-2,99$ & 2,00 & 0 & 1,01 & 2,22 & $-0,208$ & 33,33 & 18,12 & 12 \\
13 & 0 & 0 & 0 & 0 & $-1,51$ & 0,89 & $-1,04$ & 0 & 1,24 & $-0,028$ & 55,56 & 12,40 & 7 \\
$\mathbf{1 4}$ & 0 & 0 & 0 & 0 & 0 & 0 & $-0,79$ & 0 & 1,51 & 0,120 & 77,78 & 6,99 & 2 \\
15 & 0 & 0 & 0 & 0 & $-1,03$ & 0 & $-1,07$ & 0 & 1,59 & $-0,023$ & 66,67 & 9,70 & 4 \\
16 & 0 & 1,33 & 0 & 1,18 & 0 & 0,91 & $-0,93$ & $-0,85$ & 0,82 & 0,247 & 33,33 & 18,04 & 11 \\
17 & $-1,63$ & 0 & 0 & 0 & $-1,78$ & 0 & $-1,24$ & 0 & 1,40 & $-0,298$ & 55,56 & 12,53 & 9 \\
18 & $-2,27$ & 0 & 0 & 0 & $-1,35$ & 1,12 & 0 & 0 & 1,34 & $-0,038$ & 55,56 & 12,49 & 8 \\
\hline Total $^{*}$ & $\mathbf{8 9}$ & $\mathbf{9 7}$ & $\mathbf{1 0 7}$ & $\mathbf{1 0 9}$ & $\mathbf{1 2 3}$ & $\mathbf{1 1 5}$ & $\mathbf{1 2 5}$ & $\mathbf{1 3 0}$ & $\mathbf{1 4 9}$ & & & & \\
\hline
\end{tabular}

P1 à P9 = parcelas(classes de d e h), ${ }^{*} n^{o}$ de posições do tronco amostrados em cada parcela, $\mathrm{P}^{\mathrm{ns}}=\mathrm{n}^{\mathrm{o}}$ de parcelas em que o teste Dunnett foi não significativo, $\mathrm{EMD}=$ erro médio Dunnett, UPM = único porcentual médio.

Na Figura 2, o modelo 14 melhor descreve o taper que o modelo 1, expressivamente, em toda a extensão do tronco. Inclusive com tendência neiloidal, bem característico na parte basal deste, o que não se observa haver com o emprego do modelo 1. Assim, dentre 18 modelos de taper avaliados, pode-se inferir que o modelo 14, do tipo expoente-forma, é a melhor opção para quantificar multiprodutos da madeira de Eucalyptus urophylla e Eucalyptus grandis no nordeste da Bahia, com idades variando entre 5 a 7 anos.

Os resultados dos modelos selecionados neste trabalho são bem melhores aos obtidos em alguns trabalhos feitos no Brasil, onde $r\left(y_{i} \hat{y}_{i}\right)$ e $s\left(y i \hat{y}_{i}\right)$ variaram de 95 a $97 \%$ e de 6,8 a $11,4 \%$, respectivamente. Neste sentido, considerando Figueiredo Filho et al. (1996) e Assis et al. (2001), que utilizaram dados de Pinus taeda, notase que repetiu a superioridade do modelo segmentado de Clark et al. (1991) frente ao de Max \& Burkhart (1976). Já, Silva et al. (2011), trabalhando com dados de Pinus caribea var. hondurensis, obtiveram resultados favoráveis ao modelo de Baldwin Jr \& Feduccia (1991) frente aos modelos de Garay (1979), Demaerschalk (1971) e de Ormerod (1973).
Leite et al. (2006), trabalhando com dados de Virola surinamensis, verificaram superioridade do modelo de Garay (1979) frente aos modelos de Demaerschalk (1971) e de Baldwin Jr \& Feduccia (1991). Ainda, Soares et al. (2011), trabalhando com diferentes espécies nativas brasileiras, obtiveram superioridade do modelo de Baldwin Jr \& Feduccia (1991) frente aos modelos de Demaerschalk (1971) e de Ormerod (1973).

Diante disso, em conformidade com os resultados obtidos neste trabalho, verifica-se que os modelos de Demaerschalk e Ormerod são inadequados para a descrição do taper de Eucalyptus urophylla e Eucalyptus grandis. Por outro lado, notam-se bons resultados obtidos pelos modelos bio-matemáticos de Guimarães \& Leite (1992) e o modelo 12 (desenvolvido neste trabalho). Também, nota-se que o modelo segmentado de Clark et al. (1991) é bem superior ao de Max \& Burkhart (1976). Observa-se, ainda, que os melhores resultados foram obtidos pela maioria dos modelos expoente-forma, dando boas evidências quanto ao seu potencial para quantificar multiprodutos madeireiros em povoamentos florestais brasileiros. 
a

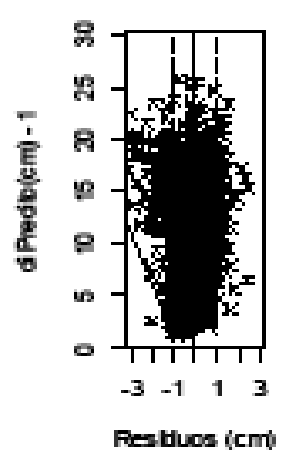

$\theta$

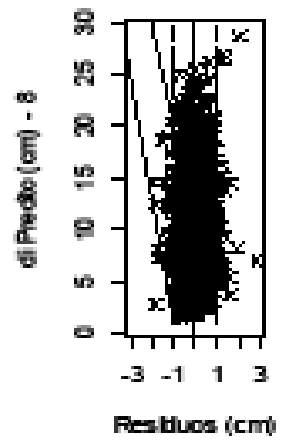

I

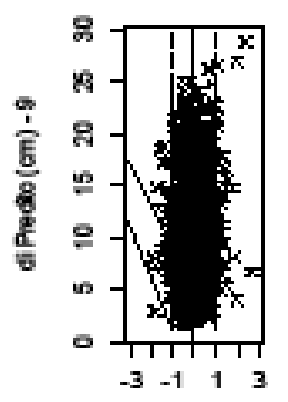

m

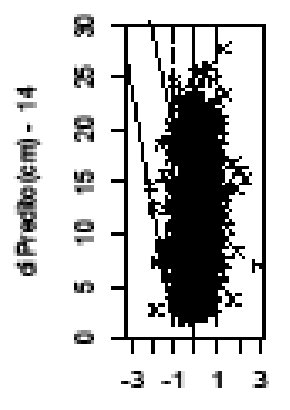

Res bluos (cm) b

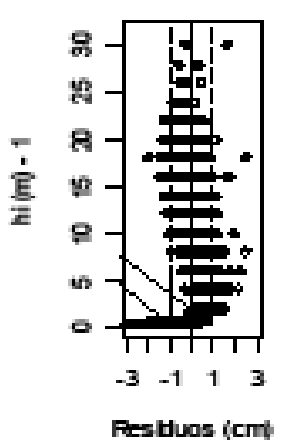

$\mathbf{f}$

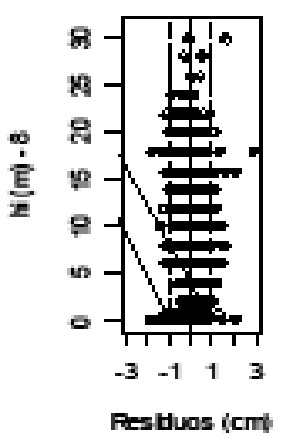

J

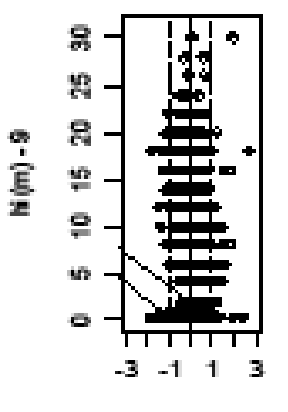

n

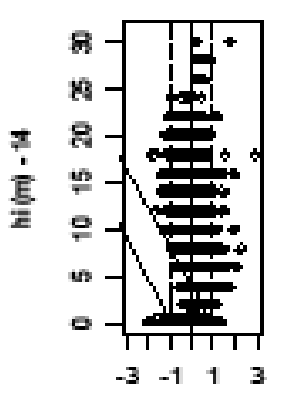

Restuuas (cm)
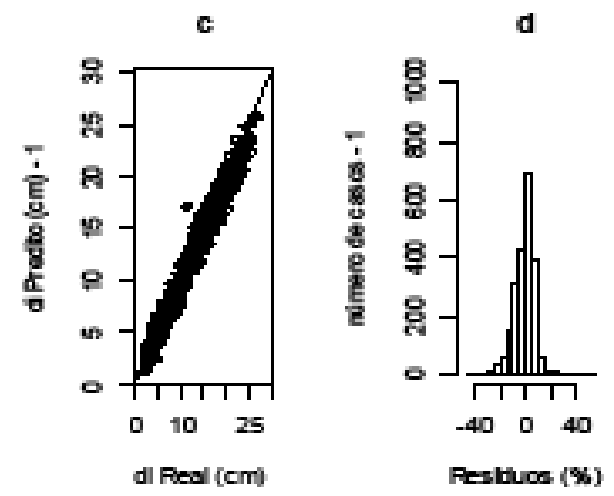

$\mathbf{h}$
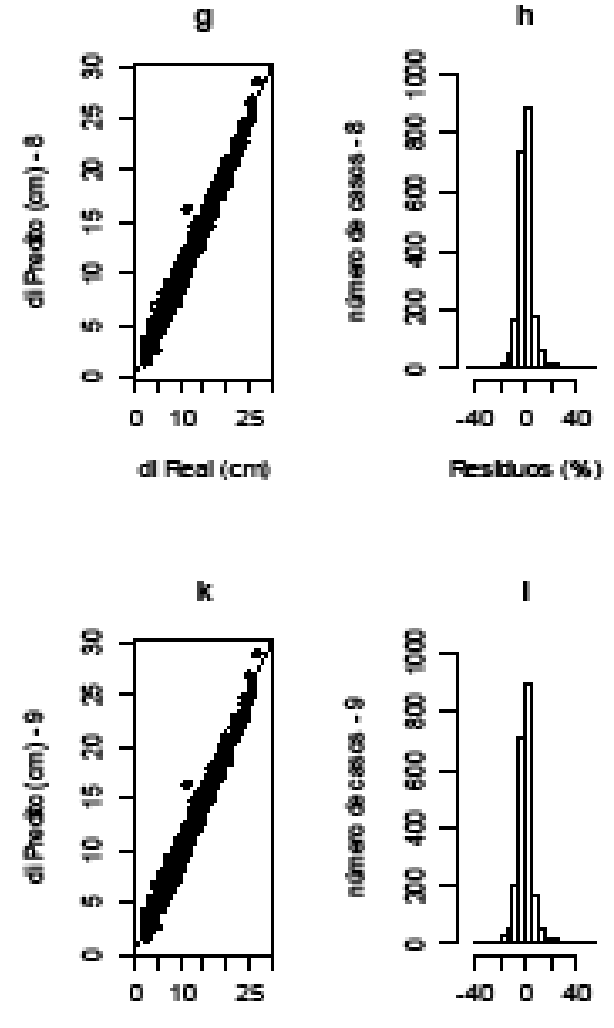

di Real (cm)

o
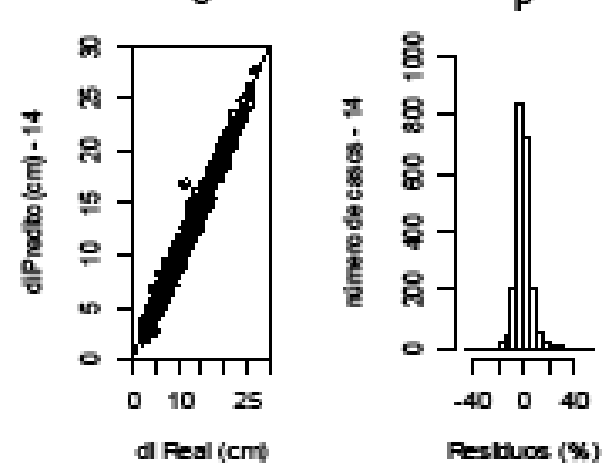

Figura 1. Distribuição de resíduos para os modelos 8,9 e 14, incluindo-se o modelo 1 por ser à forma usual de descrever o taper de árvores no Brasil. 
(a)

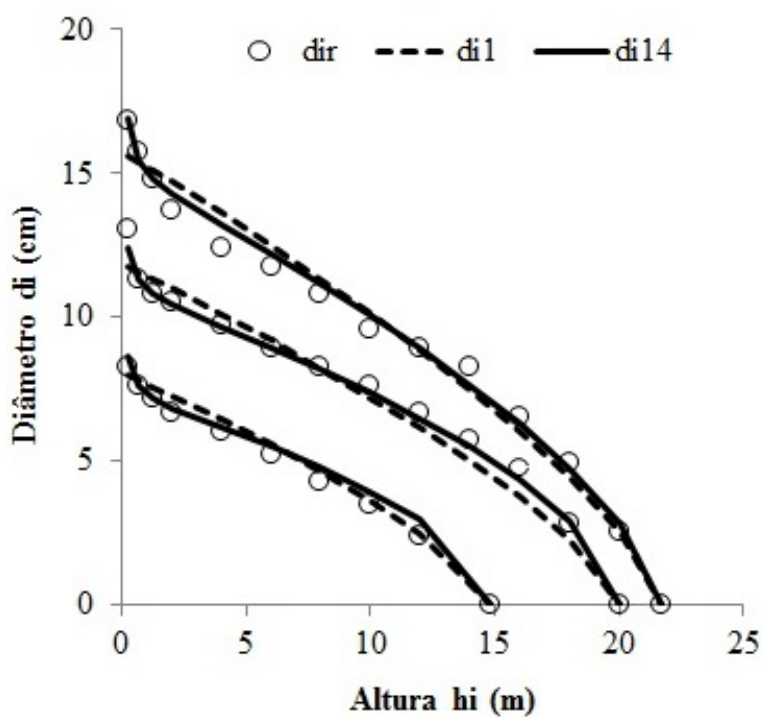

(b)

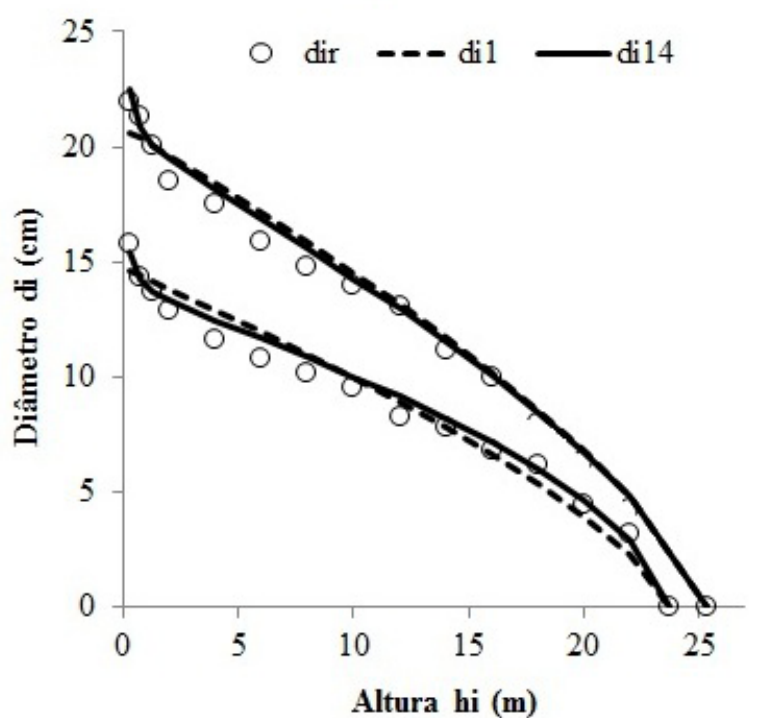

Figura 2. Perfil real (dir) e predito obtidos com os modelos 1 (di1) e 14 (di14) para uma árvore selecionada ao acaso nas classes de diâmetro 1, 2 e 4 (a), 3 e 5 (b).

\section{Conclusões}

Para descrever o perfil do fuste de Eucalyptus urophylla e Eucalyptus grandis no nordeste da Bahia, observou-se que o modelo de Demaerschalk é melhor que o de Ormerod e o modelo 14, do tipo expoenteforma, é o mais adequado de um total de dezoito modelos de taper avaliados.

Os modelos de taper expoente-forma de Muhairwe e de Kozak tem bom potencial para uso no Brasil, sendo os modelos de taper expoentes-forma superiores aos modelos de taper simples, segmentados e sigmoides avaliados neste trabalho. O modelo de taper segmentado de Clark et al. é melhor que o de Max e Burkhart e o modelo de taper 12 é melhor dentre os modelos biomatemáticos avaliados.

\section{Agradecimentos}

Ao professor da UFT, Engenheiro Florestal Renato da Silva Vieira por, gentilmente, fazer as traduções para o inglês do Título, Resumo e Termos para Indexação.

\section{Referências}

AHRENS, S.; HOLBERT, D. Uma função para forma de tronco e volume de Pinus taeda L. In: Boletim de Pesquisa Florestal, unidade regional de pesquisa florestal Centro-Sul, EMBRAPA, v. 3, p. $37-68,1981$.
ANDRADE, V.C.L.; LEITE, H.G. Uso da geometria analítica para descrever o taper e quantificar o volume de árvores individuais. Revista Árvore, Viçosa, MG, v. 25, n.4, p.481-486, 2001.

ANDRADE, V. C. L. Um método para descrever o perfil do tronco em árvores de eucalipto utilizando geometria analítica. 2001. $74 \mathrm{f}$. Dissertação (Mestrado em Ciência Florestal) - Universidade Federal de Viçosa, Viçosa, MG, 2001.

ANGElO, H.; CASTRO, L. H. R.; HOSOKAWA, R. T.; KIRCHNER, F. F. Análise de componentes principais e função spline para definir a forma do tronco de pinus tropicais. Floresta, Curitiba, v. 25, n. $1-2$, p. 55-67, 1997.

ASSIS, A. L.; SCOLFORO, J. R. S.; MELLO, J. M.; Acerbi Júnior, F. W.; Oliveira, A. D. Comparação de modelos polinomiais segmentados e não-segmentados na estimativa de diâmetros e volumes ao longo do fuste de Pinus taeda. Cerne, Lavras, v. 7, n. 1, p. 20-40, 2001.

BALDWIN JUNIOR, V. C.; FEDUCCIA, D. P. Compatible Treevolume and upper-stem diameter equations for plantation Loblolly Pines in the West Gulf region. South. Journal Applied Forestry, v. 15, n. 2, p. 92-97, 1991.

BANZATTO, D. A.; KRONKA, S. N. Experimentação agrícola. 4. ed. Jaboticabal: FUNEP, 2006. 237 p.

CAMPOS, J. C. C.; RIBEIRO, J. C. Avaliação de dois modelos de taper em árvores de Pinus patula. Revista Árvore, Viçosa, MG, v. 6, n. 2, p. 140-149, 1982.

CHICHORRO, J. F.; RESENDE, J. L. P.; LEITE, H. G. Equações de volume e de taper para quantificar multiprodutos da madeira em Floresta Atlântica. Revista Árvore, Viçosa, MG, v. 27, n. 6, p. 799-809, 2003.

CLARK, A.; SOUTER, R. A.; SCHLAEGEL, B. E. Stem profile equations for Southern tree species. Asheville: Southeastern Forest Experiment Station, 1991. 113 p. 
DEMAERSCHALK, J. P. An integrated system for the estimation of tree taper and volume. 1971. 57 f. (Mestrado) - University of British Columbia, Vancouver, Canada, 1971.

FAVAlESSA, C. M.; UBIAlli, J. A.; CALDEIRA, S. F.; DRESCHER, R. Equações de sortimentos para Tectona grandis na região centro-sul de Mato Grosso. Pesquisa Florestal Brasileira, Colombo, v. 32, n. 72, p. 389 - 399, 2012.

FIGUEIREDO FILHO, A.; BORDERS, B. E.; HITCH, K. L. Taper equations for Pinus taeda in Southern Brazil. Forest Ecology and Management, Amsterdan, v. 83, n. 1, p. 39-46, 1996.

FISCHER, F.; SCOLFORO, J. R.; ACERBI JUNIOR, F. W.; MELLO, J. M.; MAESTRI, R. Exatidão dos modelos polinomiais não-segmentados e das razões entre volumes para representar o perfil do tronco de Pinus taeda. Ciência Florestal, Santa Maria, RS, v. 11, n. 1, p. 167-188, 2001.

FRIEDL, R. A. Dinâmica e prognose da forma dos fustes em povoamentos plantados de Araucaria angustifolia (Bert.) $\mathbf{O}$. Ktze. 1989. 167 f. Dissertação (Mestrado em Ciências Florestais) - Universidade Federal do Paraná, Curitiba.

GARAY, L. A taper model for entire stemprofile including buttressing. Seattle : Institute of Forest Products, College of Forest Resources, University of Washington, 1979. 64 p. (Tropical forest utilization system, 8. Contribution, 36).

GOULDING, C. J.; MURRAY, J. C. Polynomial taper equations that are compatible with tree volume equations. New Zealand Journal of Forest Science, Rotorua, v. 5, n. 3, p. 313-322, 1976.

GUIMARÃES, D. P.; LEITE, H. G. Um novo modelo para descrever o perfil do tronco. Revista Árvore, Viçosa, MG, v. 16, n. 2, p. 170 $180,1992$.

HRADETZKY, J. Analyse und interpretation statistisher abränger keiten. (Biometrische Beiträge zu aktuellen forschungs projekten). Baden: Württemberg Mitteilungen der FVA, 1976. 146 p. (Abt. Biometric und Informatik, 21).

KOZAK, A. A variable-exponent taper equation. Canadian Journal of Forest Research, Ontario, v. 18, p. 1363-1368, 1988.

KOZAK, A.; MUNRO, D. D.; SMITH, J. G. H. Taper function and their application in forest inventory. Forestry Chronicle, Quebec, v. 45, n. 4 , p. $278-283,1969$.

LANSSANOVA, L. R.; UBIALLI, J. A.; ARCE, J. E.; PELISSARI, A. L.; FAVALESSA, C. M. C.; DRESCHER, R. Avaliação de funções de afilamento para a estimativa de diâmetro de espécies florestais comerciais do bioma amazônico mato-grossense. Floresta, Curitiba, v. 43, n. 2, p. 215 - 224, 2013.

LEE, W.-K.; SEO, J. H.; SON, Y. M.; LEE, K. H.; VON GADOW, K. Modeling stem profiles for Pinus densiflora in Korea. Forest Ecology and Management, Amsterdan, p. 69-77, 2003.

LEITE, H. G.; GAMA, J. R. V.; CRUZ, J. P.; SOUZA, A. L. Função de afilamento para Virola surinamensis (Roll.) Warb. Revista Árvore, Viçosa, MG, v. 30, n. 1, p. 99-106, 2006.

LEITE, H. G.; OLIVEIRA-NETO, R. R.; MONTE, M. A.; FARDIN, L.; ALCANTARA, A. M.; BINOTI, M. L. M. S.; CASTRO, R. V. O. Modelo de afilamento de cerne de Tectona grandis L.f. Scientia Forestalis, Piracicaba, v. 39, n. 89, p. 53-59, 2011.
MAX, T.A.; BURKHART, H. E. Segmented polynomial regression applied to taper equations. Forest Science, Lawrence, v. 22, n. 3 p. 283-289, 1976.

McTAGUE, J. P.; BATISTA, J. L. F.; STEINER, L. H. Equações de volume total, volume comercial e forma de tronco para plantações de Eucalyptus nos Estados de São Paulo e Rio de Janeiro. Revista do IPEF, Piracicaba, n. 41/42, p. 56-63, 1989.

MENDONÇA, A. R. de; SILVA, G. F. da; OLIVEIRA, J. T. da S.; NOGUEIRA, G. S.; ASSIS, A. L. de. Avaliação de funções de afilamento visando a otimização de fustes de Eucalyptus sp. para multiprodutos. Cerne, Lavras, MG, v. 13, n. 1, p. 71-82, 2007.

MUHAIRWE, C. K. Taper equations for Eucalyptus pilularis and Eucalyptus grandis for the north coast in New South Wales, Australia. Forest Ecology and Management, Amsterdam, v. 113, p. 251-269, 1999.

NEWNHAM, R. M. A Variable-form taper function. Ontario: Petawawa National Forestry Institute, Forestry Canada, 1988. 33 p. (Information Report, PI-X-83).

ORMEROD, D. W. A simple bole model. Forestry Chronicle, Quebec, v. 49, n. 3, p. 136-8, 1973.

PÉLLICO NETO, S. As curvas relativas contínuas de forma de Preußner para o sortimento dos fustes de espécies florestais. Cerne, Lavras, v. 1, n. 1, p. 17-27, 1994.

PIMENTEL-GOMES, F. Curso de estatística experimental. 15. ed. Piracicaba: FEALQ, 2009. 451 p.

PIRES, L. M.; CALEGÁRIO, N. Ajuste de modelos estocásticos lineares e não lineares para a descrição do perfil longitudinal de árvores. Revista Árvore, Viçosa, MG, v. 31, n. 5, p. 845 - 852, 2007.

SCHNEIDER, P. R.; FINGER, C. A. G., KLEIN, J. E. M. Forma de tronco e sortimentos de madeira de Eucalyptus grandis Maiden. para o Estado do Rio Grande do Sul. Ciência Florestal, Santa Maria, RS, v. 7, n. 1, p. 85-91, 1996.

SCHNEIDER, P. R. Forma de tronco e sortimentos para Pinus elliottii Engelm., da Floresta Nacional de Passo Fundo Rio Grande do Sul. Acta Forest Brasil, Curitiba, n. 1, p. 43-64, 1986.

SCHÖEPFER,W. Automatisierung des Massen, Sorten und Wertberechnung stenender Waldbestande Schriftenreihe Bad. [S.1.]: Wurtt-Forstl., Não paginado.1966.

SILVA, F.; DALLA CORTE, A. P.; SANQUETTA, C. R. Equações de afilamento para descrever o volume total do fuste de Pinus caribaea var. hondurensis na região do Triângulo Mineiro. Scientia Forestalis, Piracicaba, v. 39, n. 91, p. 367-376, 2011.

SILVA, J. A. Funções de forma dos troncos de Pinus taeda, Picea excelsa, Abies alba e Pinus sylvestris. In: SEMINÁRIO SOBRE ATUALIDADES E PERSPECTIVAS FLORESTAIS: o uso de funções de forma de tronco em estudos de volumetria de espécies florestais, 5., 1982, Curitiba. Anais... Curitiba: EMBRAPA-URPFCS, 1982. p. 29-45. (EMBRAPA-URPFCS. Documentos, 9).

SOARES, C. P. B.; MARTINS, F. B.; LEITE-JR, H. U.; SILVA, G. F.; FIGUEIREDO, L. T. M. Equações hipsométricas, volumétricas e de taper para onze espécies nativas. Revista Árvore, Viçosa, MG, v. 35, n. 5, p. 1039-1051, 2011.

SOUZA, C. A. M.; CHASSOT, T.; FINGER, C. A. G.; SCHNEIDER, P. R.; FLEIG, F. D. Modelos de afilamento para o sortimento do fuste de Pinus taeda L. Ciência Rural, Santa Maria, RS, v. 38, n. 9, dez, p. 2506-2511, 2008. 
